\title{
Thermal Cycling Toughness and Strength Estimation of Cryogenic Filled Tank
}

\author{
Dmitry Uglanov ${ }^{1, a}$, Dmitry Sarmin ${ }^{1}$, Alina Akulova ${ }^{1}$, Daria Aksenova ${ }^{1}$ and Roman Panshin ${ }^{1}$ \\ ${ }^{1}$ Samara University, Department of Heat Engineering and Heat Engines, 443086, Moskovskoye shosse, 34, Samara, \\ Russia
}

\begin{abstract}
At present, technologies based on liquid natural gas (LNG) using start to find a wide use. They also demand technologies of working fluid storage and transportation in cryogenic liquid condition. The working fluid will be used after its regasification from cryogenic liquid condition. When the gas cylinder is filled with gas, there is a danger of exceeding the temperature stresses in the cylinder walls. For the study, there is a method for calculating unsteady thermal conductivity for studying the temperature stresses in the wall of a gas cylinder during its interaction with an evaporating cryogen. This method is used for determining the thermoelastic stresses of the cryogenic filled tank. The CAE- calculation of the strength of the cryogenic filled tank is also done.
\end{abstract}

\section{Introduction}

Economic, exhibited and special requirements to gas and cryogenic liquids storage require creation of more perfect designs [1]. These requirements include abilities to endure high pressure, decreasing of the working fluid leakage, satisfaction of the accelerated fuelling requirement, meeting the safety requirement and having required durability.

Usually working fluids for technological and special purposes are stored in gaseous compressed form in high pressure tanks or in a liquid form with cryogenic temperatures.

For example, in case of natural gas using working fluid is filled in a tank to a 20 pressure of MPa and more. Fuelling is accomplished in the gas-filling stations by compressing. The time of the fuelling is equal to several minutes and is followed by tank heating which decreases the durability of the tank and may cause an under fuelling or increase the fuelling length. Fuelling by compressing require significant energy consumption.

At present, technologies based on liquid natural gas (LNG) using start to find a wide use [2-6]. There are also technologies of working fluid storage and transportation in cryogenic liquid condition. The working fluid will be used after its regasification from cryogenic liquid condition.

From aforementioned it is followed that it will be objectively useful and well-timed to develop and create universal equipment able to satisfy existing technologies of cryogenic liquid and compressed gases use.

For example, in gas-filling compressor stations it will be appropriate to combine the presently used tank filling method of compression with fuelling from cryogenic liquid condition.

\footnotetext{
${ }^{\mathrm{a}}$ Corresponding author : dmitry.uglanov@mail.ru
} 


\section{The design of the cryogenic filled tank}

The peculiarity of the cryogenic filled tank (Fig. 1), for example, for natural gas $[7,8]$ is that it can be refuelled to operating pressure (20-40 MPa), or liquefied natural gas (LNG). In the latter case, the cryogen is poured into an inner vessel, outside of which there is a multi-layer thermal insulation between it and the inner wall of the cylinder, which is necessary to prevent convection currents and to protect against heat stroke. At the same time, the heat exchange between the wall of the CNG balloon is caused only by the thermal conductivity of the gaseous methane located in the annular gas cavity.

Parameters of the working fluid in the tank was calculated by special methods [8]. It was shown that the heating of the liquid phase, its evaporation and redistribution of liquid and gaseous fractions is carried out in a relatively long period of time (up to two days). After this time, the parameters of the working fluid come to the equilibrium state of the entire balloon, with the equilibrium temperature reaching $-80 \ldots-100^{\circ} \mathrm{C}$, and the pressure does not exceed $2 \ldots 3 \mathrm{MPa}$.

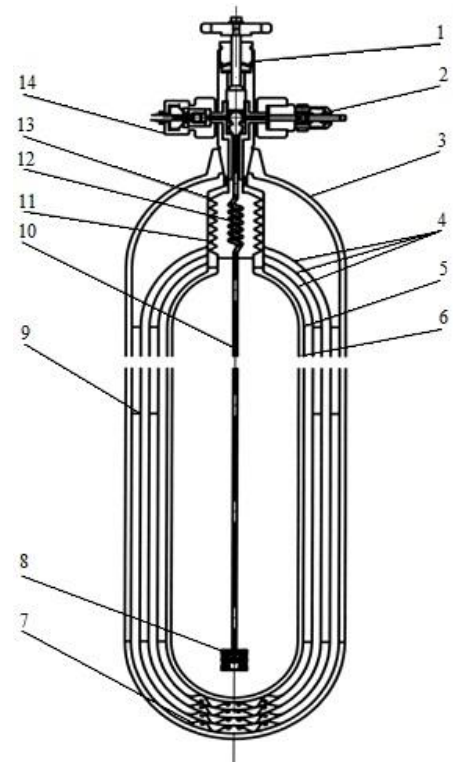

Figure 1. Cryogenic filled tank (CFT).

1 - cutoff valve; 2 - safety valve; 3 - tank; 4 - insulation; 5 - insulation of the inner volume; 6 - inner volume; 7 supporting space ring; 8 - filter; 9 - radial space rings; 10 - barrel; 11 - feeding tube; 12 - coil; 13 - bellows; 14 - flanged socket

Such parameters allow expecting small stresses in the tank wall, which increases the service life of the cylinder. Despite the fact that the problems of thermophysics in relation to the balloon can be considered to be solved in a certain amount, the issues of assessing the stress levels, and, accordingly, the thermal cycle strength requires a separate study. In the present article the solution of the problem of determination of stresses in the balloon wall in the conditions of its interaction with the evaporating cryogen is given.

\section{The calculation of the strength of the cryogenic filled tank}

The problem of determining the thermoelastic stresses is reduced to a joint solution of the strength problem and the problem of transient heat conduction. To solve this problem, the method of calculating transient heat conduction was used to study the temperature stresses [9]. The possibility of representing the problems of thermoelastic stresses simple and reliable functional operators makes it relatively easy to determine the fields of temperature stresses for any time-varying thermal effects. 
After the tank is filled with a cryogen, the liquid begins to evaporate, and the evaporated fraction of the cryoproduct is redistributed along the layers of the gas cavity. The relative radius $p=\frac{r}{R}$ for the balloon wall with internal $R_{1}$ and outer $R_{2}$ radius is introduced. Then the differential equation of transient heat conduction inside the balloon wall under mixed boundary conditions of the third and second kind in dimensionless coordinates is given to the form of:

$$
\begin{gathered}
\frac{\partial T}{\partial F_{O}}=\frac{\partial^{2} T}{\partial p^{2}}+\frac{1}{p} \cdot \frac{\partial T}{\partial p}, \\
{[T(p, F o)]_{F o-0}=T_{0} .}
\end{gathered}
$$

$T_{o}=T_{o}(c)=$ const - the initial wall temperature.

$$
\begin{gathered}
{\left[\frac{\partial T}{\partial p}-B i \cdot T(p, B i)\right]_{p=1}=-B i \cdot \varphi_{2}(F o)} \\
\left(\frac{\partial T}{\partial p}\right)_{p=k}=\frac{R_{1}}{\lambda} \cdot \varphi_{1}(F o) .
\end{gathered}
$$

Here $F_{O}=a \cdot \tau / R_{1}^{2} ; B i=\alpha \cdot R_{1} / \lambda ; k=R_{2} / R_{1}>1$.

After the corresponding transformations [3], the relative excess wall temperature for the cooling case under consideration will be expressed as a ratio:

$$
\theta(p, F o, B i, k)=\frac{T_{c p}-T(p, F o)}{T_{c p}-T_{0}}=D(B i, k) \cdot\left[\frac{(k-1) \cdot B i+2}{B i}-\frac{(k-p)^{2}}{k-1}\right] \cdot \exp \left[-A(B i, k) \cdot F_{o}\right]
$$

Tangential and radial stresses are found by the formulas with taking into account [3] in a cylindrical wall:

$$
\begin{aligned}
& \sigma_{\varphi}=\frac{\beta \cdot E}{1-v} \cdot \frac{1}{p^{2}} \times\left[\frac{p^{2}+1^{k}}{k^{2}-1} \int_{1}^{k} T(p, F o) p d p-T(p, F o) p^{2}\right] ; \\
& \sigma_{\varphi}=\frac{\beta \cdot E}{1-v} \cdot \frac{1}{p^{2}}\left[\frac{p^{2}-1^{k}}{k^{2}-1} \int_{1}^{k} T(p, F o) p d p-\int_{1}^{k} T(p, F o) p d p\right] .
\end{aligned}
$$

If we substitute the value of the current local temperature $\mathrm{T}$ (r, Fo) obtained from expression (3) into formulas (4), (5), then the dimensionless stresses:

$$
\begin{aligned}
& \overline{\sigma_{\varphi}}=\frac{(1-v) \cdot \sigma_{\varphi}}{\beta \cdot E \cdot\left(T_{c p}-T_{0}\right)} \\
& \overline{\sigma_{p}}=\frac{(1-v) \cdot \sigma p}{\beta \cdot E \cdot\left(T_{c p}-T_{0}\right)} .
\end{aligned}
$$

These equations are defined by the formulas:

$$
\begin{aligned}
& \overline{\sigma_{\varphi}}=-D(B i, k) \cdot N(p, k) \cdot \exp [-A(B i, k) \cdot F o] \\
& \overline{\sigma_{p}}=-D(B i, k) \cdot M(p, k) \cdot \exp [-A(B i, k) \cdot F o] .
\end{aligned}
$$

The values of $D(B i, k)$ and $A(B i, k)$ are calculated from the relations:

$$
\begin{gathered}
A(B i, k)=\frac{10 B i[B i \cdot(k-1)(k+3)+12]}{(k-1)\left[B i^{2} \cdot(k-1)^{2} \cdot(11 k+5)+10 B i \cdot(k-1) \cdot(5 k+3)+60(k+1)\right]} \\
D(B i, k)=2,5 \cdot \omega \cdot B i[B i \cdot(k-1) \cdot(5 k+3)+12 \cdot(k+1)] \\
\omega^{-1}=B i^{2} \cdot(k-1)^{2} \cdot(11 k+5)+10 B i \cdot(k-1) \cdot(5 k+3)+60(k+1) .
\end{gathered}
$$

Formulas (10-12) allow to determine the stresses for any values of $\mathrm{B}_{\mathrm{i}}$ and tank wall parameters $k$. By the above relations, the values of the parameters included in the calculation formulas were determined (Table 1). 
Using these parameters, the values of the relative excess wall temperatures on the inner surface of the balloon were calculated, taking into account their time dependence $\theta\left(p, F_{0}, B_{i}, k\right)$, temperature distribution in the balloon wall $T\left(p, F_{0}\right)$ as a function of time, and also radial and the tangential stresses $\sigma_{p}(p), \sigma_{\varphi}(p)$ in the tank wall. The stresses created by the pressure of the evaporating gas (graph of the change in gas pressure with respect to time, is presented in the article "Numerical modeling of processes in a fuel balloon with cryogenic refueling with natural gas for non-drainage storage") are determined using the well-known expression 10. The results of the calculations are shown in Fig. 2. In accordance with Russian State Standard [5] the margin of safety is assumed to be $n=2$. Analysis of the obtained data allows to conclude that during the whole gasification process the stresses in the tank wall do not exceed the tensile strength, for example, for the tank BK-6601-400 TU, made of steel $45, \sigma_{T}=890 \mathrm{MPa}$. Thus, the stresses in the tank wall in this case are much lower, that is, by an order of magnitude than when refueled by compression in comparison with, $\sigma_{T}$.

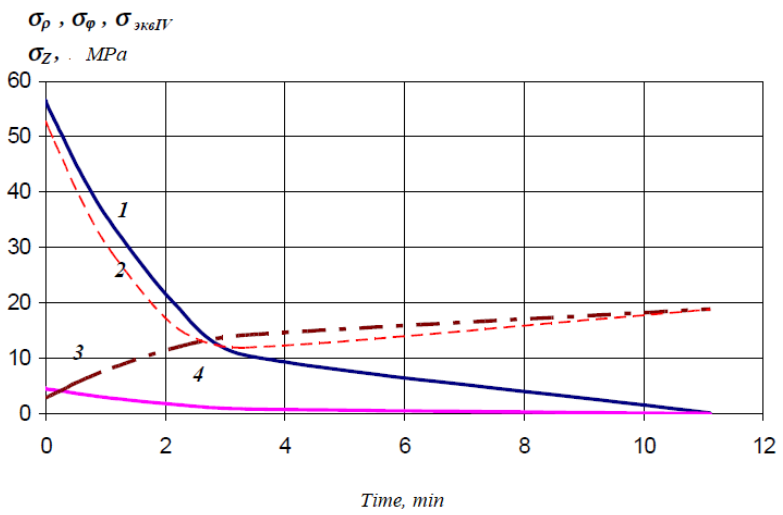

Figure 2. The graph of the change in the thermal radial $\sigma_{p}(1)$, the equivalent stresses $\sigma_{e q I V}(2)$, the stresses generated by the pressure of the evaporating gas (3) and the tangential stresses $\sigma_{\varphi}$ in the wall of the cylinder (4).

Table 1. Calculation parameters, formulas and values.

\begin{tabular}{|c|c|}
\hline Parameter & Value \\
\hline $\mathrm{R}_{1}, \mathrm{~mm}$ & 100 \\
\hline $\mathrm{R}_{2}, \mathrm{~mm}$ & 106 \\
\hline $\mathrm{K}=\mathrm{R}_{1} / \mathrm{R}_{2}$ & 1,06 \\
\hline$\lambda, \mathrm{W} /(\mathrm{m} \cdot \mathrm{K})$ & 16,3 \\
\hline$\alpha=\lambda / \mathrm{p} \cdot \mathrm{c}, \mathrm{m}^{2} / \mathrm{s}$ & $0,8510-5$ \\
\hline $\mathrm{F}_{0}=\alpha \cdot \tau R_{1}^{2}$ & $0,00085 \cdot \tau$ \\
\hline$\alpha, \mathrm{W} /\left(\mathrm{m}^{2} \cdot \mathrm{K}\right)$ & 22,9 \\
\hline $\mathrm{B}_{\mathrm{i}}=\alpha \cdot \mathrm{R}_{1} / \lambda$ & 0,013 \\
\hline$B\left(B_{i}, k\right)$ & 0,066 \\
\hline$D\left(B_{i}, k\right)$ & 0,068 \\
\hline$A\left(B_{i}, k\right)$ & 2,27 \\
\hline
\end{tabular}




$$
\begin{gathered}
\sigma_{z}=\frac{p_{g} \cdot D_{2}}{2\left(R_{2}-R_{1}\right)} ; \\
\sigma_{e q I V}=\sqrt{\sigma_{z}^{2}+\sigma_{p}{ }^{2}+\sigma_{\varphi}{ }^{2}-\left(\sigma_{z} \sigma_{p}+\sigma_{\varphi} \sigma_{p}+\sigma_{z} \sigma_{k}\right.} ; \\
\sigma_{e q I V} \leq[\sigma] ; \\
{[\sigma]=\frac{\sigma_{T}}{n} .}
\end{gathered}
$$

If we take into account the practice of using the reservoir as a fuel tank for a car, even taking into account the occasional full gasification of LNG to the parameters of fuelling by compression, it can be argued that the cylinder resource is increased at least three times.
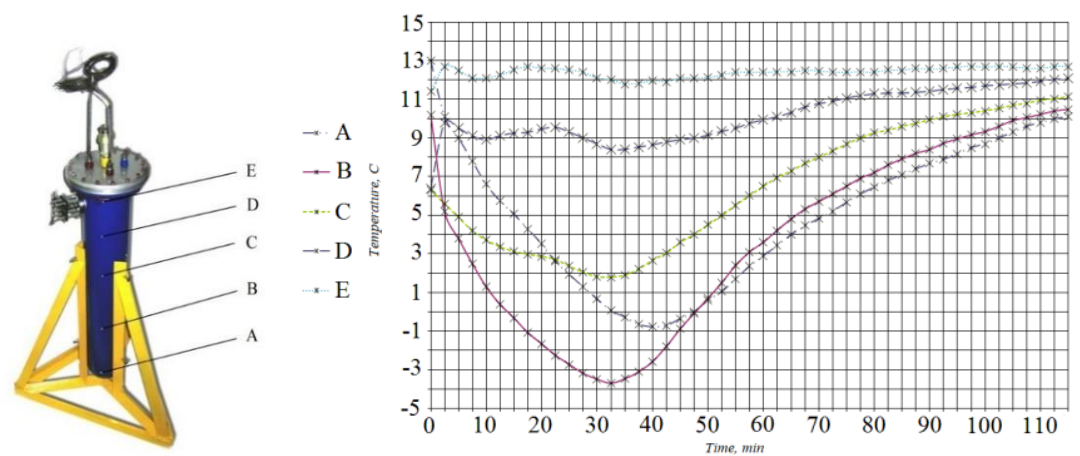

Figure 3. The distribution of the cylinder wall temperature in different sections, depending on the operating time on the flow rate.

\section{Investigation of the temperature state of the cryogenic filled tank wall}

Since one of the main functions of the internal thermal insulation of CFT is to prevent a thermal shock from the cryoproduct to the wall of the balloon, then the temperature of the CFT wall (in time) will be an important indication of this.

The temperature of the cylinder wall in different sections as a function of time was recorded by the Micron - M $7604 \mathrm{~F}$ thermal imager and is shown in Figure 4.

Measurements showed that the maximum change in wall temperature during the experiment was $120 \mathrm{~min}$. has reached $19^{\circ} \mathrm{C}$ (from $+14{ }^{\circ} \mathrm{C}$ to $-4{ }^{\circ} \mathrm{C}$ ), and it occurred for 30 minutes from the moment of hermetic sealing of the balloon, after which the temperature values in different sections equalized (Figure 3).

On the height of the balloon (vertical position) at the beginning of the process, the minimum temperature is observed in the middle of the balloon $\left(+6^{\circ} \mathrm{C}\right)$, at the end of the process - at the bottom of the balloon $\left(+9^{\circ} \mathrm{C}\right)$.

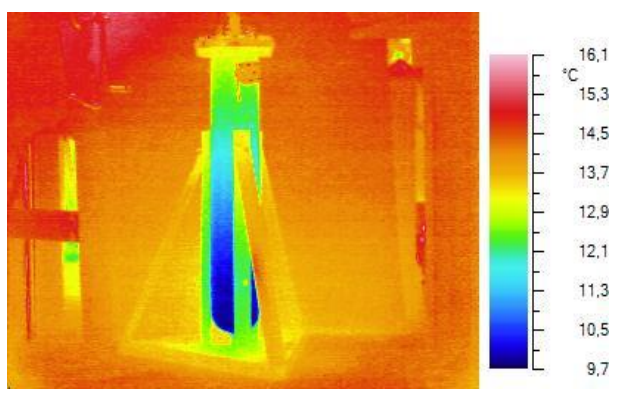

Figure 4. Initial and final state of the cylinder in the operating mode 
The main conclusion is that the temperature level itself does not exceed $20^{\circ} \mathrm{C}$, in height, and the rate of change of the gradient along the altitude $\Delta \mathrm{T}_{\mathrm{H}}$ is no more than $0.5 \mathrm{deg} / \mathrm{min}$.

The temperature gradient over the wall thickness of $\delta \approx 5 \mathrm{~mm}$ is not more than $0,05^{\circ} \mathrm{C}$ (calculations for the solution of the problem of non-stationary thermal conductivity of the system). The thermograms of the initial and final state of the cylinder wall temperature are shown in Fig. 4.

\section{The CAE - calculation of the strength of the cryogenic filled tank}

In the course of the research, a numerical assessment of the temperature influence on the elements of the prototype of the cryogenic refueling tank with the help of ANSYS SAE system was carried out.

It was created using ANSYS Meshing grid model design. By setting the grid type and cell sizes for each element of the model, a grid model acceptable for calculation was obtained. To calculate the unevenly heated cylinder, which is in the elastic-plastic state, its temperature-loaded depending on the distance to the bottom of the cylinder on the basis of earlier experimental studies (Figure 2). Upon completion of the calculations, stress fields for all elements of the model of the BKZ sample and its margin of safety are constructed. Control of the magnitude of the displacements allows to check the General plausibility of the calculations. Stresses, safety margins and displacements of all model elements are shown in figures 5.

Consider a cylindrical vessel (Figure 6) which is under the action of an internal pressure p. Radius of cylinder R, thickness $\mathrm{h}$.

The axial component of the pressure forces will be equal to the product of the pressure $p$ by the area of the projection of the surface, on a plane perpendicular to the given axis:

Thus, we obtain:

$$
P=\pi R^{2} p
$$

$$
\begin{aligned}
\sigma_{m} 2 \pi R h & =\pi R^{2} p \\
\sigma_{m} & =\frac{p R}{2 h} .
\end{aligned}
$$

For a cylinder $\rho_{m}=\infty, \rho_{t}=R$. Therefore, from Laplace's formula we find:

those. The circumferential stress is twice the meridional.

$$
\begin{aligned}
& \frac{\sigma_{\mathrm{m}}}{\infty}+\frac{\sigma_{\mathrm{t}}}{\mathrm{R}}=\frac{\mathrm{P}}{\mathrm{h}} ; \\
& \sigma_{t}=\frac{p R}{h} .
\end{aligned}
$$

For the sphere $\rho_{m}=R, \rho_{t}=R, \sigma_{m}=\sigma_{t}=\sigma$. Therefore, from Laplace's formula we find:

$$
\begin{gathered}
\frac{\sigma}{R}+\frac{\sigma}{R}=\frac{P}{h} ; \\
\sigma=\frac{p R}{2 h} .
\end{gathered}
$$
(14):

We cut off the part of the cylinder by the cross section (Figure 7), the equilibrium equation for it

$$
\sigma_{m} 2 \pi R h=P
$$

The shell, as a rule, is made of plastic materials, so we will use the third theory of limiting stresses.

$$
\begin{aligned}
& \sigma_{e q I I I}^{\max } \leq[\sigma] ; \\
& \sigma_{\text {eqIII }}^{\max }=\sigma_{1}-\sigma_{3} .
\end{aligned}
$$

The ABCD element, isolated from the cylindrical shell, is in a biaxial stress state (Figure 8): $\sigma_{1}=$ $\sigma_{t}, \sigma_{2}=\sigma_{m}, \sigma_{3}=0$

Equivalent stress for the cylindrical part:

$$
\sigma_{\text {eqIII }}^{\max }=\sigma_{1}-\sigma_{3}=\sigma_{t}-0=\frac{p R}{h} .
$$

For the spherical part of the shell, we find the equivalent voltage similarly:

Thus, for a cylindrical shell part:

$$
\sigma_{\text {eqIII }}^{\max }=\sigma_{1}-\sigma_{3}=\sigma-0=\frac{p R}{2 h} .
$$


For the spherical part of the shell:

$$
\frac{p K}{h} \leq[\sigma]
$$

For the whole shell:

$$
\frac{p R}{2 h} \leq[\sigma]
$$

$$
\frac{p R}{h} \leq[\sigma] .
$$

Since the outer shell of the cylinder is made of steel 30XMA, then for the ultimate strength we take the yield strength for this steel $\sigma_{t}=392 M P a$. From (27) we express h and take $\sigma_{\tau}=[\sigma]$. For $p$ we take $p_{\text {max }}=15 M P a$.

We take the wall thickness of $0.005 \mathrm{~m}$.

$$
h=\frac{p R_{6}}{\sigma_{\tau}}=\frac{15 \times 10^{6} \times 0,13}{392 \times 10^{6}}=0,0049 M .
$$
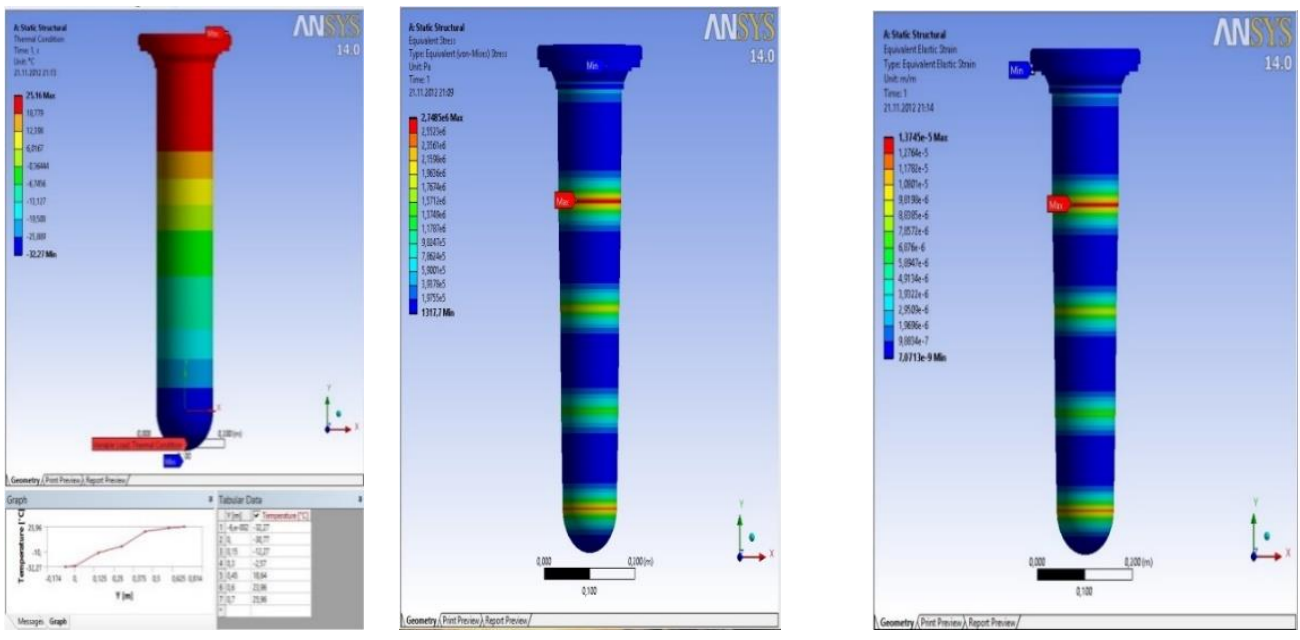

Figure 5. The graph of the change in the thermal radial (1), the equivalent stresses (2), the stresses generated by the pressure of the evaporating.

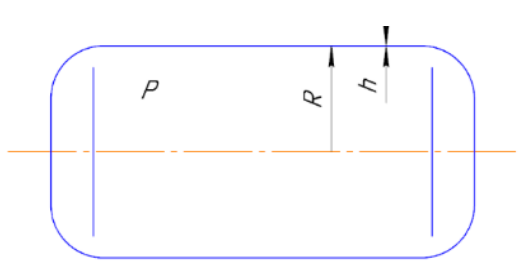

Figure 6. Cylindrical vessel under the action of internal pressure $p$

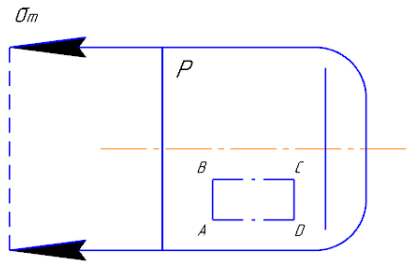

Figure 7. Cross-sectioned part of the cylinder.

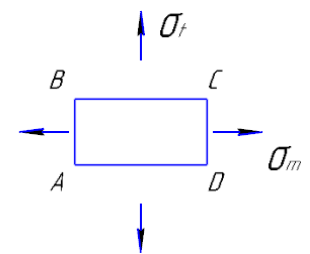

Figure 8. Isolated element from a cylindrical shell, in a biaxial stress state

We perform the verification calculation for strength:

$$
\begin{gathered}
\frac{p R_{b}}{h}=\frac{15 \times 10^{6} \times 0.13}{0.005}=390 M P a \\
390 M P a<[392 M P a]
\end{gathered}
$$

The condition of strength is observed.

\section{Conclusions}

From the result of the calculation shows that the maximum local stresses in the structure from thermal stresses occurring when filling the concert hall with cryogenic products does not exceed $2.8 \mathrm{MPa}$, 
which satisfies the conditions of strength and for a lot less compared to the stresses arising in cylinder running compression (pressure $15 \mathrm{MPa}$ ), it indicates significant potential to increase the resource of the container. The tank resource could be increased at least three times compared to standard tanks.

From the result of the calculation it can be seen that the maximum local voltage in the structure from the thermal stresses arising when filling the cryogenic filled tank with the cryoproduct does not exceed 2.8 MPa, which satisfies the strength conditions.

Compared with tanks filled with compression (pressure $15 \mathrm{MPa}$ ). This indicates a significant potential for increasing the life of the balloon.

\section{References}

1. S.-J. Jeon, B.-M. Jin, Y.-J. Kim, Consistent thermal analysis procedure of LNG storage Tank, Structural Engineering and Mechanics. 25, 4 (2007)

2. B. Adouane, W. de Jong, J.P. van Buijtenen, G. Witteveen, Fuel-NOx emissions reduction during the combustion of LCV gas in an air staged Winnox-TUD combustor, Applied Thermal Engineering, 5, 30(8) (2010)

3. A.K. Sen, G. Litak, B.-F. Yao, G.-X. Li, Analysis of pressure fluctuations in a natural gas engine under lean burn conditions, Applied Thermal Engineering, 4, 30(8) (2010)

4. Q.Y. Li, Y.L. Ju, Design and analysis of liquefaction process for offshore associated gas resources, Applied Thermal Engineering, 8, 30(8) (2010)

5. Kakumoto, Y., Koyamatsu, Y., Shiota, A., Qudaih, Y., Mitani, Y. Application of Geographic Information System to Power Distribution System Analysis, Energy Procedia, 6, 100 (2016)

6. Burtsev, S.A., Karpenko, A.P., Leontiev, A.I. A method for distributed production of liquefied natural gas at gas-distribution stations, High Temperature, 4, 54 (4), (2016)

7. Patent RU2163699.

8. E.V. Blagin, A.I. Dovgyallo, S.O. Nekrasova, D.V. Sarmin, D.A. Uglanov, Applied Thermal Engineering Estimation of the energy efficiency of cryogenic filled tank using in different system and devices, 8, 101 (2016)

9. P. V. Tsoi, Methods of calculation of heat and mass transfer problems (Moscow: Energoatomizdat, 1984) 